\title{
Size-sorting dust grains in the surface layers of protoplanetary disks
}

\author{
C. P. Dullemond ${ }^{1}$ and C. Dominik ${ }^{2,3}$ \\ 1 Max Planck Institut für Astronomie, Königstuhl 17, 69117 Heidelberg, Germany \\ e-mail: dullemon@mpia.de \\ 2 Sterrenkundig Instituut Anton Pannekoek, Kruislaan 403, 1098 SJ Amsterdam, The Netherlands \\ e-mail: dominik@science.uva.nl \\ 3 Department of Astrophysics, Radboud University Nijmegen, PO Box 9010, 6500 GL Nijmegen, The Netherlands \\ Received 7 March 2008 / Accepted 17 April 2008
}

\section{ABSTRACT}

\begin{abstract}
Context. The shape of dust emission features measured from protoplanetary disks contains information about the typical size of the dust particles residing in these disks. A flattened $10 \mu \mathrm{m}$ silicate feature is often interpreted as proof that grain growth has taken place, while a pointy feature is taken as evidence for the pristine nature of the dust.

Aims. We wish to investigate what the effect of dust sedimentation is on the observed $10 \mu \mathrm{m}$ feature and how this may affect the interpretation of the observations.

Methods. Using a combination of modeling tools, we simulated the sedimentation of a dust grain size distribution in an axisymmetric 2-D model of a turbulent protoplanetary disk, and we used a radiative transfer program to compute the resulting spectra.

Results. We find that the sedimentation can turn a flat feature into a pointy one, but only to a limited degree and for a very limited set of particle size distributions. If the distribution is too strongly dominated by small grains, then the feature is pointy even before sedimentation. If the distribution is too strongly dominated by big grains, the sedimentation will not be enough to cause the feature to be pointy. Only if we have a bimodal size distribution, i.e. a very small grain population and a bigger grain population, do we find that the transformation from a flat to a pointy feature upon dust sedimentation is strong. However, our model shows that, if sedimentation is the sole reason for the variety of silicate feature strengths observed in protoplanetary disks, then we would expect to find a correlation such that disks with weak mid- to far-infrared excess have a stronger $10 \mu \mathrm{m}$ silicate feature than disks with a strong mid- to far-infrared excess. If this is contrary to what is observed, then this would indicate that sedimentation cannot be the main reason for the variety of $10 \mu \mathrm{m}$ silicate features observed in protoplanetary disks.
\end{abstract}

Key words. radiative transfer - accretion, accretion disks - dust, extinction

\section{Introduction}

The protoplanetary disks surrounding brown dwarfs, $\mathrm{T}$ Tauri stars, and Herbig Ae/Be stars have displayed a rich variety of dust emission features in their infrared spectra. These features have been studied in great detail with the Infrared Space Observatory (ISO; e.g. Meeus et al. 2001; Bouwman 2001), with ground-based instruments (van Boekel et al. 2003; Honda et al. 2003) and with the Spitzer Space Telescope (e.g. Apai et al. 2005; Kessler-Silacci et al. 2006; Furlan et al. 2006). While the wavelength and width of these dust emission features reveals the composition of the dust grains and whether they have undergone thermal processing or not, the shape and strength of the features betrays the dominant size of the emitting grains. Infrared spectroscopy is therefore a powerful tool for studying the onset of grain growth, which is believed to be the very initial step toward planet formation.

A drawback of this kind of analysis is that it only probes the very tenuous "superheated" surface layers of the disk. This layer, which is produced by the irradiation of the disk by the central star (Calvet et al. 1991; Chiang \& Goldreich 1997), is very optically thin and has a much higher temperature than the disk interior. For these reasons the dust in this layer typically produce strong emission features. Although this surface layer contains only a minuscule part of the mass of the disk, it is responsible for about half of the infrared luminosity of the disk (Chiang \& Goldreich 1997) and entirely dominates the generation of dust emission features. This raises the concern about how representative this layer is for the entire disk, and whether changes to dust properties observed in this layer are in any way related to changes deeper in the disk, where most of the mass resides and important processes such as planet formation take place. Of particular concern is that dust tends to sediment toward the midplane until an equilibrium between sedimentation and vertical turbulent mixing is established (Dubrulle et al. 1995). Large grains have a lower surface-to-mass ratio and are therefore less prone to friction with the gas than small grains. They therefore settle more deeply into the disk interior before an equilibrium between sedimentation and turbulent mixing is established. Small grains, however, stay afloat much higher up in the photosphere of the disk, and since they dominate the opacity anyway, they maintain the photospheric disk height at a higher level. The bigger grains therefore settle out of the photosphere, into the deep optically thick regions of the disk. They are therefore filtered out of sight.

The question therefore arises: could it be that the dust in this surface layer is very atypical compared to the bulk of the disk deeper in the optically thick parts of the disk? If this is the case, what will we learn from studying the infrared spectra from these disks? This paper is not the first to raise this question. It has also been noted by e.g. van Boekel et al. (2003), and Sicilia-Aguilar et al. (2007) have invoked the potential effect of big grains sinking out of the surface layer in their analysis of Spitzer spectra of a sample of pre-main-sequence stars. Kessler-Silacci et al. (2006) 
speculate that, by looking at different wavelength ranges, one looks deeper into the disk toward regions of larger grains. However, no quantitive study of this problem has been presented yet. A related problem has, however, been studied recently by Dullemond et al. (2007). That paper showed that dust sedimentation can enhance the infrared features from polycyclic aromatic hydrocarbons (PAHs) in the disk, because the sedimentation causes the thermal dust grains to sink below the photosphere held up by the PAHs.

In the present paper we wish to study the effect of sedimentation on the shape of the $10 \mu \mathrm{m}$ feature of silicate dust. This solid state infrared emission feature, seen in the majority of protoplanetary disks, has often been used as a probe of grain size (e.g. van Boekel et al. 2005; Schegerer et al. 2006). A strong and pointy $10 \mu \mathrm{m}$ feature is typical of grains smaller than about $1 \mu \mathrm{m}$ in radius, while a weak and flattened feature indicates the presence of grains between $2 \mu \mathrm{m}$ and $4 \mu \mathrm{m}$, clearly larger than the average grain size in the interstellar medium. Van Boekel et al. (2003) showed that not only are such variations in feature shape and strength observed in protoplanetary disks, but indeed also the correlation between shape and strength expected from the laboratory measurements is observed. We are aware that the interpretation of the feature shape in terms of grain size is still under debate, as porosity and fractal structure of the aggregates may influence the absorption/emission cross section (Voshchinnikov et al. 2006; Min et al. 2005). Moreover, Juhasz et al. (A\&A, submitted) has shown that high signal-to-noise is needed to interpret these features in the first place. But the fact that observations appear to confirm the predicted trend gives some hope that the method of interpretation of these features is reasonable.

Many attempts have been made to link the shape and strength of the $10 \mu \mathrm{m}$ feature to the evolution of the disk as a whole. But not even the direction of this trend is clear. Does the a flat, broad feature indicate a more developed disk by showing that larger grains have been produced in the disk? This is the most common interpretation in the literature, and it keeps the assumption alive that growth from submicron grains to micron-sized grains is a process that happens slowly in the course of a million years. However, such a trivial, direct connection seems rather unlikely, given the speed at which coagulation can proceed and produce large dust grains (Dullemond \& Dominik 2005). In view of dust settling, the exact opposite relation with the developmental state of the disk is possible as well. Assuming a steady-state size distribution and an equilibrium state between settling and turbulent mixing, the amount of large grains still present in the surface layer can be a simple effect of the strength of turbulent vertical mixing in the disk. As turbulence and gas densities die down toward the end of the disk evolution, large grains would tend to disappear from the observer's view and result in pointy features for evolved disks. For interpreting this type of spectroscopy and linking it to the evolutionary state of a disk, it is vital to understand if simple settling can indeed filter out large grains efficiently. To establish or disprove this possibility is the core purpose of this paper.

To study the effect of sedimentation on the $10 \mu \mathrm{m}$ silicate feature shape, we used the same setup as in the above mentioned paper on PAHs (Dullemond et al. 2007). We set up a disk structure in the same way as described in Dullemond \& Dominik (2004b). We then assumed some grain size distribution throughout the disk and solve the time-dependent 1-D vertical dust sedimentation-mixing equations at each radial grid point for each grain size. Finally we used the Monte Carlo continuum radiative transfer code RADMC to produce the infrared spectra,

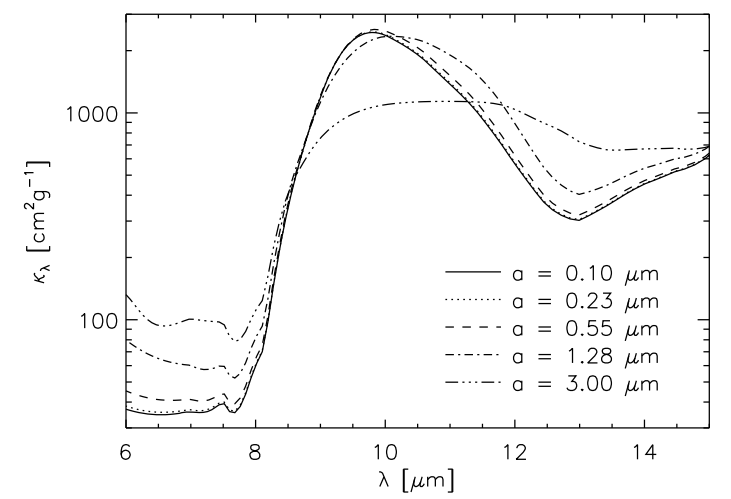

Fig. 1. The absorption cross-section in $\mathrm{cm}^{2} /$ gram-of-dust in the $N$-band range for the dust we use in our model for various spherical grain radii. For grain radii smaller than $0.1 \mu \mathrm{m}$, the opacity does not change much from the opacity of $0.1 \mu \mathrm{m}$ grains in this wavelength domain.

focusing on the region between $6 \mu \mathrm{m}$ and $15 \mu \mathrm{m}$. We then analyzed these results and figured out if dust sedimentation can modify the feature shape. In particular we investigated if sedimentation can turn a flat feature indicative of large grains into a pointy feature indicative of small grains.

The structure of this paper is as follows. In Sect. 2 we discuss the opacities we use and how we construct grain size distributions. We then discuss the model in Sect. 3 and the results in Sect. 4. Finally we conclude in Sect. 5.

\section{Opacities and grain size distributions}

The results of our analysis may depend on the precise details of the opacities and grain size distributions used. Therefore we discuss them here in some detail. We used $n$ and $k$ optical constants from the Jena database for $\mathrm{Mg} \mathrm{Fe} \mathrm{SiO}_{4}$. We produced the absorption cross-section using a Mie calculation and a specific weight of $\xi=3.6 \mathrm{~g} / \mathrm{cm}^{3}$. We did not include scattering in the radiative transfer calculations. With these Mie calculations we obtained an opacity for a grain of a specific radius $a$.

To model a grain size distribution we must choose a discrete set of grain radii between the minimum radius $a_{\text {min }}$ and the maximum radius $a_{\max }$ of the distribution. Throughout this paper we choose $a_{\min }=0.001 \mu \mathrm{m}$ and $a_{\max }=3 \mu \mathrm{m}$. In our model we always choose the sampling points to be logarithmically divided between these bounds, such that we have linear spacing in $\log (a)$. Figure 1 shows the opacities for a limited set of grain sizes, just to show the shape change occurring when one goes from small ( $a \lesssim 0.3 \mu \mathrm{m}$ ) to big $a \gtrsim 2 \mu \mathrm{m}$ grains. It should be noted that flat-topped (boxy) features can also be produced by certain mixtures of small crystalline silicate grains (e.g. Honda et al. 2003). The flatness of the feature is then due to the summing of a number of narrow crystalline peaks lined up so that, taken together, they look like a broad flat-topped feature of an amorphous large grain. High-resolution and -sensitivity measurements, however, would be able to distinguish these two scenarios by identifying the slight bumpy shape of the flat-topped feature in the crystalline silicate scenario, where the bumps are at the known locations of the peaks of enstatite and forsterite.

To obtain the total cross section per gram of dust we need to speficy the grain size distribution function $f(a)$. We define $f(a)$ such that

$\rho_{\text {dust }}=\int_{a_{\min }}^{a_{\max }} m(a) f(a) \mathrm{d} a$ 


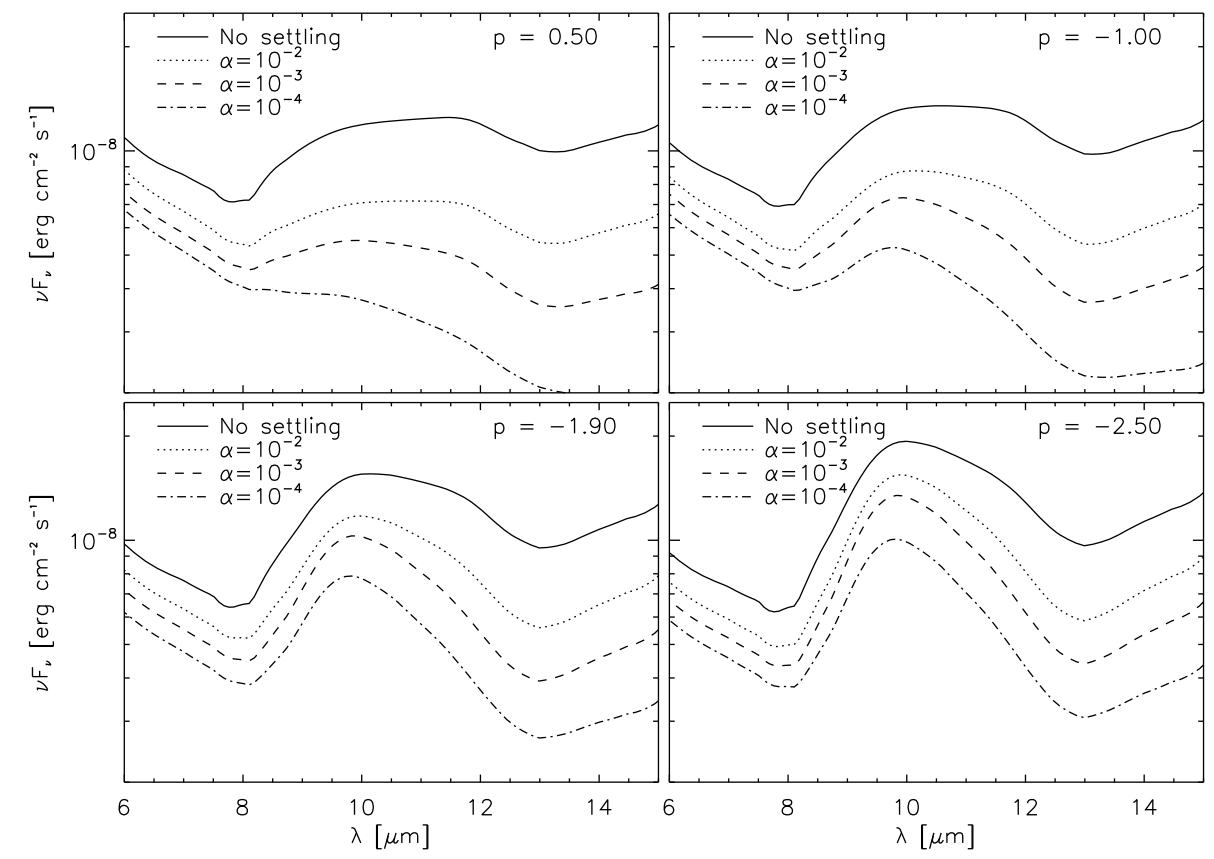

Fig. 2. Model results for the continuous size distribution with smallest size $0.001 \mu \mathrm{m}$ and the biggest size $3 \mu \mathrm{m}$. The four different panels are for four different values of the powerlaw index $p$ for the grain size. The four different lines in each panel are for no settling (top) and for settling-mixing equilibrium with three different levels of turbulence. is the total dust density in units of $\mathrm{g} / \mathrm{cm}^{3}$. Here $m(a)=(4 \pi / 3) \xi a^{3}$ with $\xi=3.6 \mathrm{~g} / \mathrm{cm}^{3}$. Throughout this paper we take a powerlaw distribution: $f(a) \propto a^{p}$. The case $p=-7 / 2$ represents the MRN distribution (Mathis et al. 1977). In terms of a size distribution in particle mass $\tilde{f}(m) \propto m^{q}$ such that $\tilde{f}(m) \mathrm{d} m=f(a) \mathrm{d} a$, one gets $q=(p-2) / 3$, so that for the MRN distribution we have $q=$ $-11 / 6$. However, from now on we shall use the $f(a)$ formalism. If we sample this distribution function with $N$ discrete $a$-values, then we can associate a dust density to each of these "bins":

$\rho_{\text {dust }}^{(i)}=m\left(a_{i}\right) a_{i} f\left(a_{i}\right) \Delta \log (a)_{i}$

where $\Delta \log (a)_{i}$ is the width of each bin in $\log (a)$ which we assume to be the same for all bins, except for the first and the last, which are only half as wide to ensure that the distribution goes exactly from $\log \left(a_{\min }\right)$ to $\log \left(a_{\max }\right)$. The total radiative cross section $\alpha_{v}$ in units of $1 / \mathrm{cm}$ is then

$\alpha_{v}=\sum_{i=1, N} \rho_{\text {dust }}^{(i)} \kappa_{v}^{(i)}$.

The total emissivity $j_{v}$ in units of $\mathrm{erg} \mathrm{s}^{-1} \mathrm{~cm}^{-3} \mathrm{~Hz}^{-1} \mathrm{ster}^{-1}$ is then

$j_{v}=\sum_{i=1, N} \rho_{\mathrm{dust}}^{(i)} \kappa_{\nu}^{(i)} B_{v}\left(T_{i}\right)$

where $T_{i}$ is the temperature of grains with size $a_{i}$. We allow the grains of different sizes to obtain different temperatures, because we assume that they are not mutually thermally coupled, nor do they have strong thermal coupling with the gas.

Because each grain size can have a different temperature, the $\alpha_{v}$ is not really a correct representation of the expected output spectrum, because the Planck functions with which the individual $\rho_{\text {dust }}^{(i)} \kappa_{v}^{(i)}$ are multiplied may be different for each $i$. The full radiative transfer calculations described later in this paper will take care of this.

\section{Model}

We start our model with making a vertical structure calculation of a disk around a Herbig Ae star with $T_{*}=10000 \mathrm{~K}$, $R_{*}=2.5 R_{\odot}, M_{*}=2.5 M_{\odot}$. The disk has an inner radius of $0.7 \mathrm{AU}$, an outer radius of $100 \mathrm{AU}$, and a surface density distribution of $\Sigma(R)=\Sigma_{0}(R / \mathrm{AU})^{-1}$ with $\Sigma_{0}=163 \mathrm{~g} / \mathrm{cm}^{2}$. We then use the procedures described in Dullemond \& Dominik (2004a) to create a self-consistent disk structure that is in vertical hydrostatic equilibrium. This procedure involves solving the radiative transfer equation in 2-D axisymmetry using a multidimensional radiative transfer program, and iterating this with the vertical hydrostatic equilibrium equation. This procedure then gives $\rho_{\text {gas }}(r, z)$, i.e. the gas density as a function of radius and vertical height above the midplane. In this procedure we assume that the gas and the dust are well-mixed and thermally coupled (i.e. $T_{\text {gas }}=T_{\text {dust }}$ ) and we assume that the dust consists of small $0.1 \mu \mathrm{m}$ size silicate grains.

Once we have this gas density distribution we insert the real dust density distribution that we wish to model, again with the same dust-to-gas ratio of 1:100. We then use the time-dependent dust settling code described in detail in Dullemond \& Dominik (2004b). This gives the density $\rho_{i}(r, z, t)$ of each dust component $i$. For a bimodal distribution we have only $i=1,2$, while for a continuous distribution we have $i=1 \ldots 20$, as we model the distribution with 20 bins. We take the time $t$ to be $1 \mathrm{Myr}$, so that we are sure that the dust has settled in a settling-mixing equilibrium.

\section{Results}

We do the above described experiment for three values of the turbulent strength: $\alpha=10^{-2}, 10^{-3}, 10^{-4}$, and for four different continuous size distributions $p=-2.5,-2.0,-1.0,0.5$. The results are shown in Fig. 2. We find that settling can make the feature more pointy, but not dramatically so. This is better seen in Fig. 4left, where we plot the well-known strength-shape diagram of van Boekel et al. (2005). This diagram shows the strength of the feature over the continuum on the $x$-axis and the normalized ratio of the fluxes at 11.3 and $9.8 \mu \mathrm{m}$ on the $y$-axis. Small grains (pointy features) appear at the lower right of the diagram, while big grains (flat features) appear at the top right. It should be noted here, however, that crystallization of the grains have an effect similar to grain growth by ending up in the top left of the diagram, but we are not concerned here about crystallization 

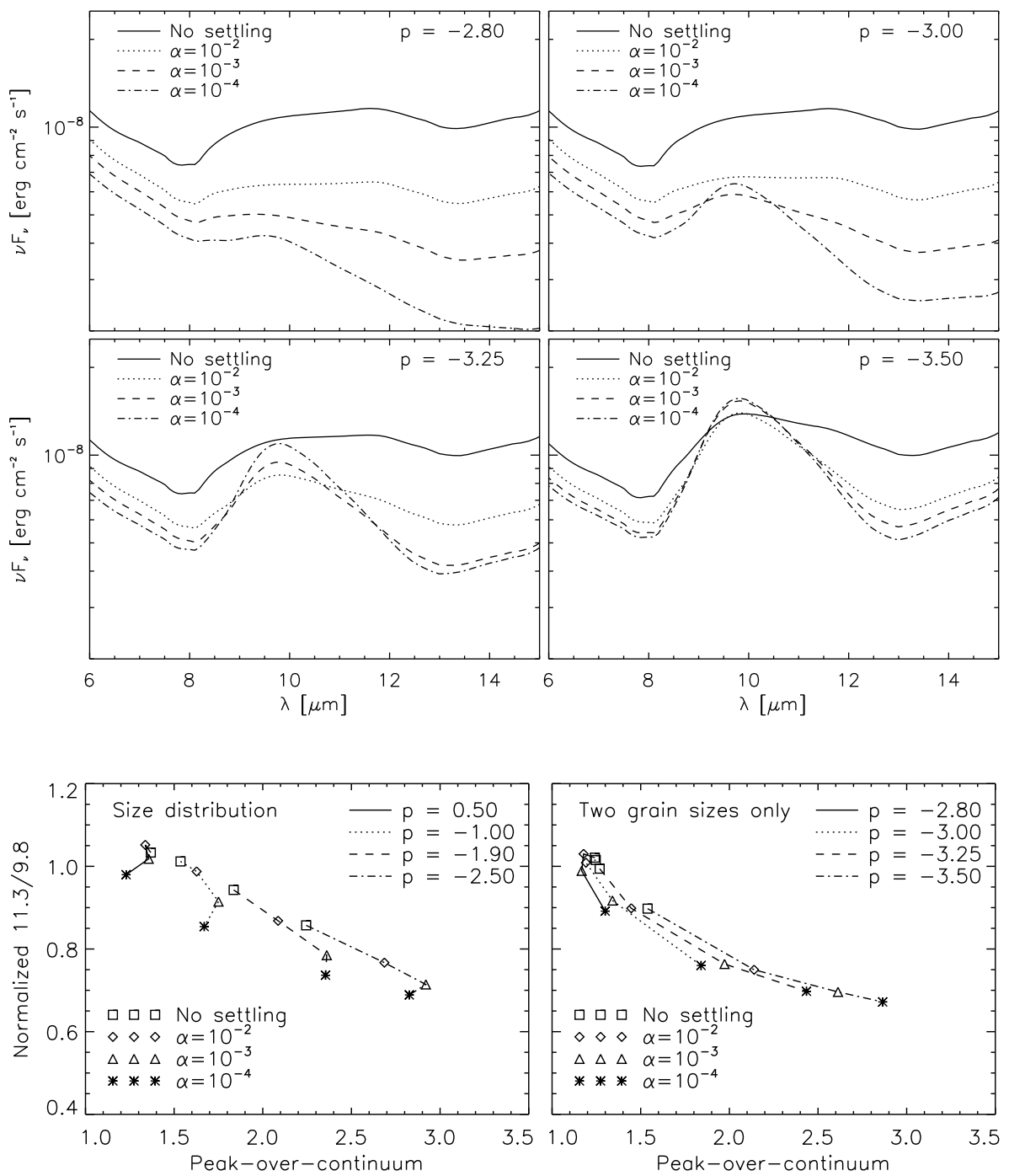

Fig. 3. As Fig. 2. Model results for the bimodal size distribution with the small grain size $0.001 \mu \mathrm{m}$ and the big grain size $3 \mu \mathrm{m}$.
Fig. 4. The feature-strength versus shape diagram of van Boekel \& Min (2005) for both the continuous distribution of sizes (left) and the bimodal size distribution (right). Models with the same size distribution are indicated by lines. The different symbols are the different levels of turbulence. now. What we do expect is that if settling causes a flat feature to be pointy, the same model for ever lower values of $\alpha$ would be seen as a movement from top left to bottom right. In the figure these models are connected by lines. One sees that the expected effect is only reasonably strong for $p=-1$ and $p=0.5$, but weak or absent for $p=-2.5$ and $p=-2$.

Now we do the same for the bimodal size distribution, in which we have only a small grain size $(0.001 \mu \mathrm{m})$ and a big one $(3.0 \mu \mathrm{m})$. Figure 3 shows that the effect of settling is very strong: a flat feature can now really be converted into a truly pointy one. For $p=-2.8$, this happens quite suddenly between $\alpha=10^{-4}$ and $\alpha=10^{-3}$, while for $p=-3.25$ this happens equally suddenly between $\alpha=10^{-3}$ and $\alpha=10^{-2}$, and for $p=-3.5$ this happens for any value of $\alpha$. This is also reflected in Fig. 4-right, where one can now clearly see the top-left to bottom-right trend predicted when going from flat to pointy. Interestingly, such a strong boosting of the small grain population has also been seen in the models with PAHs (Dullemond et al. 2007). So it appears that if we have a clearly bimodal distribution, then the settling can boost the features of the small grains (be they true grains or PAHs), but for a continuous distribution this is not as easy.

In addition to the feature-boosting effect described here, dust sedimentation is also known to reduce the mid- to far-infrared flux of a disk (Miyake \& Nakagawa 1995; Chiang et al. 2001; Dullemond \& Dominik 2004b; D'Alessio et al. 2006). To be more precise: the ratio of, say, $30 \mu \mathrm{m}$ flux over $13 \mu \mathrm{m}$ flux will decrease for decreasing level of turbulence (and hence increasing level of sedimentation). The reason for this is that the disk becomes geometrically flatter as seen in the dust continuum. The outer regions of the disk therefore capture less stellar radiation and consequently produce weaker mid- to far-infrared emission. Depending on the situation, it can even happen that the dust in outer disk regions has sunk so deeply into the disk that it resides entirely in the shadow cast by the inner disk regions (Dullemond \& Dominik 2004b). This suppresses the midto far-infrared flux even more and also makes the disk nearly invisible in scattered light. Now, if sedimentation is responsible for the boosting of small-grain features in some disks, then there should be a correlation between the feature strength/shape and the mid- to far-infrared flux. In a recent paper, Bouwman et al. (2008) plot the flux ratio $F_{30 \mu \mathrm{m}} / F_{13 \mu \mathrm{m}}$ versus $10 \mu \mathrm{m}$ featureover-continuum for a small sample of $\mathrm{T}$ Tauri stars. They find a correlation in which the disks with weak feature also have a low $F_{30 \mu \mathrm{m}} / F_{13 \mu \mathrm{m}}$. They interpret this as the effect of grain growth: as the grains grow, the $10 \mu \mathrm{m}$ silicate feature becomes weaker and flatter, and because the overall opacity of the disk goes down, so does the mid- to far-infrared flux compared to the $N$-band flux. However, in the scenario we study in this paper, in which different strengths of the $10 \mu \mathrm{m}$ feature are supposed to be due to different strength of turbulence, we would expect the opposite correlation: for low turbulence the feature becomes stronger, while the $F_{30 \mu \mathrm{m}} / F_{13 \mu \mathrm{m}}$ becomes weaker. Indeed this 

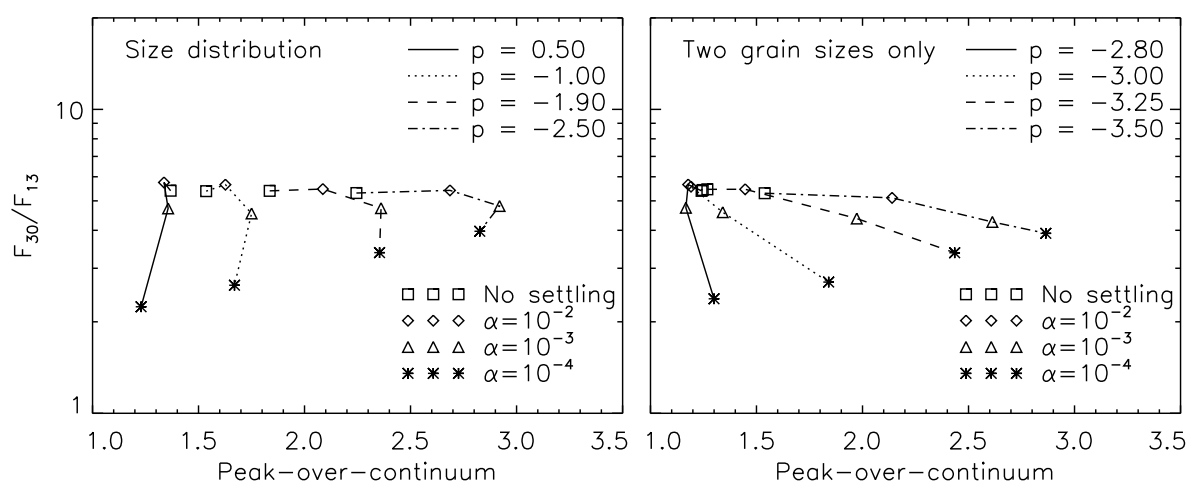

Fig. 5. The model predictions for the correlation between feature strength ( $x$-axis) and SED shape ( $y$-axis). The SED shape is the ratio of the flux (in Jy) at $30 \mu \mathrm{m}$ and at $13 \mu \mathrm{m}$. Left panel is for the continuous size distribution models. Right panel is for the bimodal size distribution models. is what comes out of our models, as can be seen in Fig. 5: a stronger feature correlates with a smaller $F_{30 \mu \mathrm{m}} / F_{13 \mu \mathrm{m}}$. For the grain size distribution this trend is rather weak (but certainly not the opposite trend), but the trend is clear for the bimodal size distribution. If the feature strength - SED shape correlation found by Boumwan et al. is confirmed on the basis of larger samples, then this would serve as clear proof that sedimentation alone cannot be held responsible for the strength or weakness of the $10 \mu \mathrm{m}$ feature.

In another recent paper, Watson et al. (2007) found a correlation between the crystallinity of the dust as deduced from Spitzer IRS spectra and the SED overall shape. Disks that are more crystalline appear to have a flatter geometry. Since there appears to be no physical reason why crystalline grains are typically smaller than amorphous ones, the sedimentation filtering process we discussed in this paper does not predict any correlation between these things to happen. The explanation for the correlation found by Watson et al. must therefore lie in something else. In principle, as mentioned before, a perfect line-up of crystalline peaks in the $9-11 \mu \mathrm{m}$ region of the spectrum may mimic a flat-topped feature from large amorphous silicates, and may affect the conclusions of Bouwman et al. (2008). But it appears that the analysis by Bouwman et al. is precise enough to exclude this possibility.

\section{Conclusion}

We have modeled the sedimentation of grains of different sizes in a disk, with particular emphasis on the $10 \mu \mathrm{m}$ silicate feature shape. We expected that sedimentation removes big grains from the surface layers, leaving the smaller grains behind, so that the spectrum will then be dominated by a pointy $10 \mu \mathrm{m}$ feature indicative of small grains, even if the initial size distribution is dominated by the flat-topped features characteristic of big $(3 \mu \mathrm{m})$ grains. We find that this is indeed the case for bimodal size distributions if the smaller grains size is chosen extremely small. In our model we have a clearly defined population of $3 \mu \mathrm{m}$ size grains and a clearly defined $\ll 0.1 \mu \mathrm{m}$ grains. The arising effect is reasonably strong because there is a large difference in the surface-to-mass ratio between these grains, and therefore a strong effect on differential settling.

On the other hand, our attempt to produce this effect with a continuous size distribution have basically failed. With a continuous (powerlaw) distribution of grains spanning the range $0.001 \mu \mathrm{m}$ all the way to $3 \mu \mathrm{m}$, we find only a very weak effect, much less than what is observed. Therefore, unless the dust size distribution is strongly bimodal distribution with just the right large and small grain sizes, this excludes sedimentation alone as the sole origin of the different $10 \mu \mathrm{m}$ silicate feature strengths and shapes seen in the spectra of protoplanetary disks. In other words, the shape and strength of the silicate feature, which originates in the surface layers of the disk, is reasonably representative of the dust hidden deep within the disk, at least for grains in the size range up to a few micron. Changes in the $10 \mu \mathrm{m}$ feature do indicate changes in the small grain population in the disk.

A similar conclusion can be made with respect to the influence of the distribution of grains on the overall geometry of the disk. A pure sedimentation model (i.e. without growth) predicts that disks with low $30 \mu \mathrm{m}$ flux over $13 \mu \mathrm{m}$ flux ratio should typically have a stronger and pointier $10 \mu \mathrm{m}$. This is most clearly expected for the bimodal size distribution. For the continuous size distribution, this correlation is expected to be weaker because of the weaker effect of sedimentation on the silicate feature shape. But at the very least such a model does not predict the opposite trend: that weak 30-over-13 flux ratio correlates with a weak feature, which is the trend observed by Bouwman et al. (2008). If this trend can be confirmed for a larger sample, this would then constitute rather strong proof that sedimentation alone cannot be held responsible for the variety in observed silicate strengths. Dust coagulation, in combination of course with sedimentation, must then play a vital role in producing the different silicate strengths and shapes observed in disks around young stars.

Acknowledgements. We wish to thank Rens Waters, Thomas Henning, and Jeroen Bouwman for very stimulating discussions.

\section{References}

Apai, D., Pascucci, I., Bouwman, J., et al. 2005, Science, 310, 834 Bouwman, J., Meeus, G., de Koter, A., et al. 2001, A\&A, 375, 950 Bouwman, J., Henning, T., Hillenbrand, L. A., et al. 2008, ArXiv e-prints, 802 Calvet, N., Patino, A., Magris, G. C., \& D'Alessio, P. 1991, ApJ, 380, 617 Chiang, E. I., \& Goldreich, P. 1997, ApJ, 490, 368

Chiang, E. I., Joung, M. K., Creech-Eakman, M. J., et al. 2001, ApJ, 547, 1077 D’Alessio, P., Calvet, N., Hartmann, L., Franco-Hernández, R., \& Servín, H. 2006, ApJ, 638, 314

Dubrulle, B., Morfill, G., \& Sterzik, M. 1995, Icarus, 114, 237

Dullemond, C. P., \& Dominik, C. 2004a, A\&A, 417, 159

Dullemond, C. P., \& Dominik, C. 2004b, A\&A, 421, 1075

Dullemond, C. P., \& Dominik, C. 2005, A\&A, 434, 971

Dullemond, C. P., Henning, T., Visser, R., et al. 2007, A\&A, 473, 457

Furlan, E., Hartmann, L., Calvet, N., et al. 2006, ApJS, 165, 568

Honda, M., Kataza, H., Okamoto, Y. K., et al. 2003, ApJ, 585, L59

Kessler-Silacci, J., Augereau, J.-C., Dullemond, C. P., et al. 2006, ApJ, 639, 275

Mathis, J. S., Rumpl, W., \& Nordsieck, K. H. 1977, ApJ, 217, 425

Meeus, G., Waters, L. B. F. M., Bouwman, J., et al. 2001, A\&A, 365, 476

Min, M., Hovenier, J. W., \& de Koter, A. 2005, A\&A, 432, 909

Miyake, K., \& Nakagawa, Y. 1995, ApJ, 441, 361

Schegerer, A., Wolf, S., Voshchinnikov, N. V., Przygodda, F., \& Kessler-Silacci, J. E. 2006, A\&A, 456, 535

Sicilia-Aguilar, A., Hartmann, L. W., Watson, D., et al. 2007, ApJ, 659, 1637 van Boekel, R., Waters, L. B. F. M., Dominik, C., et al. 2003, A\&A, 400, L21 van Boekel, R., Min, M., Waters, L. B. F. M., et al. 2005, A\&A, 437, 189

Voshchinnikov, N. V., Il'in, V. B., Henning, T., \& Dubkova, D. N. 2006, A\&A, 445,167

Watson, D. M., Leisenring, J. M., Furlan, E., et al. 2007, ArXiv e-prints, 704 